\title{
Análisis exploratorio de investigaciones sobre los motores de combustión interna que trabajan con biogás
}

\section{An exploratory analysis of existing research on internal combustion engines operating with biogas}

Jorge Eduardo Arango Gómez

Ingeniero Mecánico, magíster en Motores de Combustión Interna, magíster en Automatización. Profesor asistente de la Universidad Nacional de Colombia. Bogotá, Colombia. Contacto: jearangogo@unal.edu.co

\section{Fabio Emiro Sierra Vargas}

Ingeniero Mecánico, magíster en Automatización Industrial, doctor en Ingeniería. Profesor titular de la Universidad Nacional de Colombia. Bogotá, Colombia. Contacto:fesierrav@unal.edu.co

\section{Vladimir Silva Leal}

Ingeniero Mecánico, magíster en Ingeniería Mecánica. Investigador de la Universidad Nacional de Colombia. Bogotá, Colombia. Contacto: vsilval@unal.edu.co

Fecha de recepción: 14 de junio de 2012

Fecha de aceptación: 12 de febrero de 2013
Clasificación del artículo: revisión

Financiamiento: Universidad Nacional de Colombia

Palabras clave: motor de combustión, emisiones, relación de compresión, mezcla de gases, biogás, eficiencia.

Keywords: engine combustion, emissions, compression ratio, gas mixture, biogas, efficiency.

\section{RESUMEN}

Las metodologías y aplicaciones sobre el uso de la tecnología del biogás aparecen en el mundo en gran parte de las investigaciones científicas sobre el tema de la eficiencia, la combustión y el control de emisiones en motores de combustión interna (MCI), las cuales han venido desarrollando en un contexto histórico el mejoramiento de aspectos sociales, económicos y ambientales de Asia, Europa, África y Latinoamérica. En el presente análisis se da una mirada al estado del arte de los motores que trabajan con biogás, teniendo en cuenta su evolución y aplicaciones 
cotidianas, y se identifican algunos de los parámetros de operación y comportamiento de estos equipos que trabajan por combustión mediante ignición por chispa y encendido por compresión. Los resultados que se muestran describen una mejora considerable en su desempeño y manifiestan la importancia de continuar profundizando en la investigación sobre el uso de biogás en motores de explosión utilizados en la transformación de energía, ya que la demanda energética actual y la necesidad de optimizar su uso en el ámbito mundial se hacen vitales a la hora de pensar en los parámetros y ajuste del motor que permitan mejorar sus condiciones de desempeño.

\section{ABSTRACT}

The methodologies and applications on the use of biogas technology in the world appear in many scientific research studies on the issue of efficiency, combustion and emission control in internal combustion engines. The present analysis is a survey of current cutting-edge biogas engines. The survey includes engine development and every-day applications and also identifies some of the operating parameters and behavior of such pieces of equipment, whose operation relies on spark ignition, combustion, and compression ignition. The findings point to a considerable improvement in engine performance and also demonstrate the importance of further research on the use of biogas in internal combustion engines, which can be used in processing power e.g. energy supply for current demand and the need to optimize worldwide use of engines. This appears to be vital when thinking about the engine and its adjustable parameters in order to improve standard performance.

\section{INTRODUCCIÓN}

La implementación de mejoras tecnológicas y de control en los procesos de transformación de materias primas en la industria ha mejorado la eficiencia energética y la reducción de emisiones. La demanda de energía mundial que está en constante aumento debido al crecimiento poblacional e industrial conlleva al aumento del consumo de combustibles, hecho que ha despertado gran interés por el dominio de estos métodos alternativos tanto por combustibles como por el desarrollo de fuentes renovables de generación de energía. Sin embargo, algunas regiones del mundo presentan restricciones de energía considerables y otros países, tras la búsqueda de independencia del petróleo, buscan la forma de generar energía a partir de recursos renovables propios que reduzcan emisiones y generen beneficios mediante la implementación de mecanismos de desarrollo limpio [1].
Uno de los grandes enfoques mundiales para generación de energía es el uso del biogás, que es obtenido a partir de diferentes tipos de biomasas derivadas de actividades humanas, como son los residuos orgánicos urbanos y los productos agropecuarios, con que cuentan la mayoría de los países y en amplia disponibilidad [2]. El uso del biogás en diferentes rangos de composición se ha extendido hacia la generación de energía y transporte [3], donde su entrada se ha visto favorecida en plantas térmicas y el control de emisiones en motores de combustión interna (MCI) que contaban anteriormente con implementaciones de combustibles gaseosos [4].

El uso del biogás se justifica principalmente por la reducción de emisiones de gases de efecto de invernadero al medio ambiente, mediante la degradación confinada de residuos orgánicos y la captación controlada de productos como el metano, con efecto de invernadero 21 veces mayor al $\mathrm{CO}_{2}$; 
o mediante el uso de biomasas agrícolas, que fueron absorvedoras de $\mathrm{CO}_{2}$ durante su crecimiento (plantas), para la producción de biogases sustitutos de los combustibles fósiles, que durante la combustión restituyen al ambiente el $\mathrm{CO}_{2}$ absorbido por las biomasas generando un impacto neto leve o nulo sobre la concentración de este contaminante efecto de invernadero en el ambiente [5].

\section{APLICACIONES DEL BIOGÁS EN EL ÁMBITO MUNDIAL}

El biogás como combustible para vehículos se aplica mundialmente desde 1950, mientras que en Europa se usa para tractores [6], [7], en Brasil el objetivo es sustituir la gasolina y el gasóleo en el sector de la automoción a partir de biogás purificado y comprimido o gas natural [8].

Desde mediados de los setenta se ha resaltado la importancia cada vez mayor del biogás, no solo para uso en pequeña escala en los hogares, sino como un producto de tratamiento de residuos municipales e industriales con la fermentación anaerobia [9] - [12]. La importancia dada al biogás en los países en desarrollo deriva en investigaciones que generan avances en varias zonas de Asia y África [13].Con el aumento significativo de la producción de biogás, la utilización de técnicas para la transformación en energía mecánica con implementación en motores modificados de encendido provocado por chispa eléctrica (MEP) y de encendido por compresión (MEC) tomó gran importancia en aplicaciones directas para diferentes tipos de maquinaria [14], [15], mientras que los grandes motores diseñados específicamente para el gas estaban en la generación eléctrica.

Para la década de los ochenta, se identificaron más de 100 plantas de biogás alimentando grupos electrógenos en las explotaciones de minas en Norteamérica y se estimó un número superior en zonas rurales de Europa. Para esta época, también en Estados Unidos, muchos de los sistemas con biogás proporcionaban energía eléctrica para la operación de grandes granjas y se vendía la energía excedente a los sistemas interconectados.

En 1982, China contaba con 1100 grupos electrógenos alimentados por biogás (la mayoría de estas unidades producía entre 5 y $7 \mathrm{~kW}$ ), también se contaba con 2 plantas de biogás que proporcionaban iluminación aproximadamente a 40 hogares en poblaciones de Sri Lanka, Filipinas disponía de 8 plantas de generación en granjas de cerdos, Singapur contaba con al menos 2 plantas de energía eléctrica con biogás en grandes granjas, y Ruanda, en África central, entre 1981 y 1983 se contaba con un sistema de biogás de origen belga que proporcionaba hasta $28 \mathrm{kWh}$ diariamente en una institución rural para niños [16].

El uso de los MCI alimentados por biogás [17] ha tenido amplia acogida en los países en vía de desarrollo, donde se destacan regiones asiáticas con implementaciones principalmente sobre losMEC con potencias inferiores a $10 \mathrm{HP}$. Se estableció para el 2001 que China contaba con más de 800 motores de baja capacidad alimentados con biogás que eran utilizados principalmente en bombeo de agua para cultivos, operación de máquinas fijas en zonas rurales, en el sector agrícola para procesar arroz, trilla de granos y molienda. También muchas granjas contaban con MEP de 4 y 8 cilindros alimentados con biogás, los cuales accionaban molinos de maíz y de mezcla de diferentes materiales, produciendo hasta 100 toneladas de producto diariamente [15].

Actualmente, en Botsuana en el sur de África, se utilizan sistemas para bombeo basados en motores de $1 \mathrm{HP}$, alimentados con biogás, que entregan $4300 \mathrm{l} / \mathrm{h}$ de agua desde una profundidad de $60 \mathrm{~m}$ para 2500 animales y más de 40 familias. Por otra parte, en granjas de Filipinas, se emplean sistemas electrógenos de biogás para 
entregar $580 \mathrm{gal} / \mathrm{min}$ de agua a plantas de envasado de carne [13], [18].

En cuanto a los países de la Unión Europea, se viene ampliando el interés en la investigación sobre las características del biogás, su aplicación y operación en MCI desde el punto de vista integrado de producción energética y cuidado ambiental. Hoy en día se emplea en cogeneración para la producción de calor y electricidad, como combustible en MCI estacionarios y más recientemente como carburante en automóviles. En el mundo la tendencia actual indica que cada vez son más los países que están introduciendo tarifas favorables para la energía producida a partir de MCI alimentados por biogás. Los países europeos son un claro ejemplo y muestran un gran progreso con la directiva de la Unión Europea de obtener el $20 \%$ de su energía de fuentes renovables para el 2020. En Alemania, a finales de 2007 había más de 4000 plantas de biogás operativas que proporcionaban $1270 \mathrm{MWh}$ de electricidad y se espera que la energía producida a partir de biogás llegue a los 3000 MWh para el año 2020. En Estados Unidos se ha legislado recientemente un paquete de incentivos para las empresas que desarrollen proyectos de biogás. Un reciente estudio de la Organización de Naciones Unidas (ONU) ha indicado que para el año 2025 se habrán construido más de 100000 plantas de biogás en todo el mundo [19].

La investigación sobre la combustión del biogás mezclado con otros gases como el hidrógeno para el control de emisiones y aumento en la eficiencia de los MCI [17] se sugiere como alcance de esta revisión del estado actual sobre el uso del biogás puro y sus mezclas con otros combustibles alternativos como fuente de energía para MEP y MEC. Se identifica, por tanto, la tendencia mundial en uso de biogás puro o mezclado y su influencia sobre el comportamiento de los MCI [20], las modificaciones recomendadas sobre los parámetros operativos y de configura- ción, así como las metodologías y procedimientos de ensayo más utilizados.

\section{USO DEL BIOGÁS EN MOTORES DE COMBUSTIÓN INTERNA}

Los MCI que operan con biogás son una tecnologíade gran aplicación en el campo de la generación de energía eléctrica y térmica, a pesar de que se conoce que una mezcla estequiométrica de biogás-aire aporta en promedio alrededor del $85 \%$ de la energía de una mezcla estequiométrica diesel-aire. Sin embargo, el uso del biogás se complica un poco dada la calidad de su composición química, ya que se trata de un combustible relativamente pobre, que contiene un volumen apreciable de $\mathrm{CO}_{2}$ y contaminantes como material particulado, humedad y ácido sulfhídrico $\left(\mathrm{H}_{2} \mathrm{~S}\right)$. Esto hace necesario al menos un pretratamiento de limpieza y secado para el biogás y una selección muy cuidadosa del motor. En el mercado se pueden encontrar varios proveedores de estos motores, como también proveedores de los accesorios fundamentales como filtros, deshumidificadores, sistemas de recuperación y de disipación de calor, sistemas de control y de conexionado a la red eléctrica y proveedores de paquetes completos, adecuados a las necesidades de cada interesado y de cada motor [21]. Por esta razón, aunque la tecnología se está aplicando, la configuración de parámetros del motor no está bien definida, teniendo en cuenta que los MCI son diseñados a condiciones de operación muy diferentes a las que se someten realmente, como es el caso de motores estacionarios, para los cuales su operación en relación con sus condiciones geográficas y climáticas, influyen en su desempeño, el cual afecta directamente su eficiencia.

En el MEP se puede usar el biogás filtrado como combustible único. El MEC, al no poseer bujía, no emite chispa para hacer la explosión dentro del cilindro, en cuyo caso se debe utilizar el 
biogás mezclado con el combustible diesel, que sí explota por compresión. En el caso de MEC se ha logrado sustituir hasta el $65 \%$ del combustible diesel por biogás, sin afectar al motor ni su eficiencia [4]. La modificación general para un MEC o un MEP es la adición de un mezclador que agrega el biogás en el aire de admisión. Se han utilizado varios tipos de mezcladores de airegas, los cuales se instalan fácilmente entre el filtro y la tubería de admisión de aire; en el caso de los MEP la mezcla contiene alrededor del 15\% de biogás que corresponde a una parte de biogás por cada 6 o 7 partes de aire para MCI de mezcla estequiométrica o levemente menor para $\mathrm{MCI}$ de mezcla pobre. Una de las principales aplicaciones del biogás en los MEC es la operación alterna con biogás (operación dual), pero las modificaciones efectuadas conllevan que el motor se transforme en un MEP cuando usa biogás debido a la necesidad de adicionarle un sistema de encendido, lo que se utiliza comúnmente en motores estacionarios de baja capacidad.

En el caso de los MEC, las proporciones de biogás son menores y dependen de la forma o el tipo de conversión aplicada. Hasta el 90\% del combustible fósil puede ser remplazado con biogases, pero se hace necesario el uso de diesel como combustible piloto para encender el biogás (operación bicombustible), además pueden ajustarse en configuración dual o dedicado. En los MEC de capacidad media se mantiene el encendido por compresión mediante la adición parcial de combustible diesel, lo que obliga a efectuar modificaciones a los reglajes del gobernador de la bomba de inyección o a la exploración más detallada sobre otros parámetros que influyen en el rendimiento [22], [23].

Actualmente en plantas de generación eléctrica la eficiencia del MCI se incrementa con la potencia generada hasta alcanzar un valor cercano al 38\% para MCI de distinto tamaño y también para operación a carga parcial; sin embargo, la calidad del biogás también impacta en los rendimientos de conversión. Anterior a la aparición de los MCI de mezcla pobre, las eficiencias alcanzadas eran bastante bajas, siendo casi la mitad de las actuales.

Para el caso de los MEP que utilizan el biogás producido por diferentes países en desarrollo, se han realizado cambios en el avance de chispa y la relación de compresión, presentándose consenso sobre dichas modificaciones; sin embargo, en China, el Instituto Sichuan de maquinaria agrícola llegó a la conclusión para los MEC de que los cambios en el ritmo de inyección o en la relación de compresión del cilindro tendrían un efecto red negativo en el rendimiento cuando se operan con biogás [24].

A diferencia de los MEC, para los MEP con capacidad en el rango de 5 a $150 \mathrm{~kW}$ se han reportado reducciones sustanciales de la energía entregada operando con biogás. Para los motores con capacidad, en el rango de 150 a $1500 \mathrm{~kW}$ existe una pérdida del $10 \%$ de la eficiencia en producción indirecta de potencia mecánica al operar con biogás, aunque para aplicaciones de generación,el biogás es producido in situ. Esta pérdida carece de importancia debido a la mayor flexibilidad en el uso de la energía disponible, y al autoabastecimiento de sus necesidades energéticas diarias durante muchas temporadas del año sin utilizar combustibles fósiles [25], [17].

\subsection{Encendidos por compresión (MEC) operados con biogás}

En los MEC el sistema de inyección de combustible suministra una determinada cantidad de combustible líquido según el régimen de operación. Sin embargo, el motor aspira y comprime una mezcla de aire con biogás que ha sido preparada en el dispositivo de mezcla externo; luego, esta mezcla se enciende junto con el diesel líquido. Para operar el MEC con carga parcial se reduce 
el suministro de biogás mediante una válvula de control de gas que induce una restricción simultánea en el flujo de aire, disminuyendo la eficiencia y la presión media efectiva. Por tanto, la relación de aire/combustible debe ser la afectada y cambia con la cantidad de biogás mezclado [26] - [28]. Analizando el rendimiento de los MEC duales operados en el modo combustible diesel-gas, este alcanza valores similares al logrado utilizando solamente combustible líquido, ya que el poder calorífico del biogás es similar. El ducto de entrada y el colector de admisión de los MEC están dimensionados de tal manera que se garantice una relación combustible diesel/aire óptima para la operación bajo estas condiciones. Cuando se aspira una mezcla de aire/biogás en lugar de solamente aire, la cantidad de aire neto aspirado se reduce en la misma proporción de mezcla aire/biogás, pero el combustible diesel se reduce en función de la relación de densidades diesel/biogás, de forma que la relación de exceso de aire aumentará entre 1,2 y 1,3 con una entrada total de combustible menor que la cantidad en operación con diesel. Como resultado del exceso de aire, la máxima potencia de salida en el modo bicombustible puede ser menor que operando solamente con combustible diesel. A regímenes medio y bajo, la entrada de aire es mayor de lo necesario y permite la succión de una proporción relativamente mayor de combustible en el aire, por tanto para estos casos la potencia de salida no será significativamente menor que en operación diesel [33], [36].

Para el caso de los MEC en configuración dual, la Universidad de Antioquia analizó un MEC para generación de electricidad, operado con dieselbiogás (bicombustible), donde se ha estudiado la incidencia del sistema de mezclado y la calidad del combustible piloto en el funcionamiento. El motor fue acoplado a un generador eléctrico y evaluado a un régimen de $1800 \mathrm{rpm}$ con varios grados de carga, el biogás se ha simulado con una composición típica de $60 \%$ metano $\mathrm{CH}_{4}$ y $40 \%$ de $\mathrm{CO}_{2}$. Se han encontrado disminuciones en la eficiencia efectiva al reducir la cantidad de combustible piloto en modo dual, al igual que aumentos considerables en las emisiones de $\mathrm{CH}_{4} \mathrm{y}$ monóxido de carbono CO. La opacidad del humo no varía significativamente en modo dual, pero muestra una reducción drástica al compararla con las medidas para el motor en modo diesel. Los resultados obtenidos confirman la importancia del uso del biogás como alternativa para un impacto importante en la reducción de emisiones de efecto invernadero y como aporte a la disminución de la dependencia energética y económica de los combustibles fósiles [29].

En los MEC diesel-biogás, se ha logrado un nivel de sustitución de combustible diesel del $70 \%$ para funcionamiento continuo del MEC. Al comparar la operación en modo bicombustible al $70 \%$ de sustitución con el modo diesel, se ha observado aumento en las emisiones de $\mathrm{CH}_{4}$ (7 veces mayor) y CO ( $85 \%$ mayor), los cuales se atenúan al disminuir el nivel de sustitución. Pese a los valores altos de $\mathrm{CH}_{4}$ respecto al modo diesel, se debe recordar que en general se presenta una reducción en la emisión de $\mathrm{CH}_{4}$ a la atmósfera por la utilización del $60 \%$ en volumen que tiene el biogás en el proceso de combustión del motor [29]. Se observó también que el sistema de mezclado influye de forma significativa en la eficiencia efectiva, el nivel de sustitución, las emisiones de metano $\left(\mathrm{CH}_{4}\right)$ y monóxido de carbono $(\mathrm{CO})$ a carga parcial, mientras que la calidad del combustible piloto muestra una marcada influencia en la eficiencia y las emisiones de $\mathrm{CO}$ para cargas mayores [30].

\subsection{MEP operados con biogás}

La implementación del biogás en los MEP se da a partir de modificaciones de los que consumen gasolina y tienden a producir menos energía que cuando son operados con combustible líquido fósil. La razón de este fenómeno es una disminución en la eficiencia volumétrica en la cámara de 
combustión al ser el biogás un combustible gaseoso que ocupa una mayor porción de volumen en la mezcla aspirada por el motor. Para el caso de la gasolina, esta se entrega líquida y al vaporizarse sobre la corriente de aire en el colector de admisión, los efectos de enfriamiento generan un aumento de la densidad y, por tanto, la cantidad de mezcla aire/combustible realmente aspirada por el motor ocupa un menor volumen, afectando en menor medida el desempeño del motor [4].

La modificación de un MEP es relativamente fácil [31], esta se basa en la adición de un mezclador de aire/gas en lugar de carburador. El control del motor se realiza mediante la variación del suministro de mezcla; un aumento en la relación de compresión parece ser deseable, ya que proporciona un aumento en la eficiencia del proceso desde el punto de vista termodinámico [32], [33]. Es de esperarse un bajo consumo específico de combustible y una mayor potencia de salida teniendo en cuenta el ajuste de parámetros de operación. La modificación para la operación del MEP en el modo de biogás es permanente y debe evitarse el uso del combustible original. El ajuste del punto de ignición es importante ante la lenta velocidad de combustión del biogás aprovechando la ventaja de que este no impone ningún problema específico a encendido de sistema estándar, dando la ventaja de realizar ajustes en una gama suficientemente amplia [26], [28].

Para MCI de gran capacidad se efectuó un estudio a partir de la conversión a encendido provocado, de un MEC convencional Hino K-13CTI de $13000 \mathrm{c} / \mathrm{c}$ y 24 válvulas que opera a $1500 \mathrm{rpm}$ acoplado a un generador trifásico de 4 polos y $132 \mathrm{~kW}$ para producir electricidad a $50 \mathrm{HZ}$. La conversión consistió en la adición de un carburador de biogás para la mezcla de aire-combustible, la reducción de la relación de compresión de 16:1 a 8:1 mediante la aplicación de un separador en la cabeza del cilindro, la sustitución del sistema de inyección de combustible por un sistema de encendido por chispa y la modificación de la válvula wastegate del turbo cargador para regularla presión de sobrealimentación.

Para este estudio se varió: el coeficiente exceso de aire entre 0,9 y 1,2 , el ángulo de avance de encendido entre $50^{\circ}$ y $60^{\circ}$ antes del punto muerto superior, y el ajuste de la sobrepresión de turbocargado entre los 40 a $68 \mathrm{kPa}$. La máxima eficiencia obtenida fue de $28,6 \%$ para un exceso de aire de 1,097 , un avance de chispa de ignición de $54^{\circ}$ antes del punto muerto superior, y una sobrepresión de turbocargado de $56 \mathrm{kPa}$, con una potencia de generación de 134,2 kilovatios y unas emisiones de $\mathrm{CO}$ y óxidos de nitrógeno (NOx) de 1154 y 896 ppm, respectivamente [16]. En este régimen de funcionamiento de $1500 \mathrm{rpm}$, la eficiencia del MCI aumentó conel incremento de la presión del turbo entre 40 y $56 \mathrm{KPa}$, teniéndose un ligero acrecentamiento en las emisiones de $\mathrm{NOx}$ y $\mathrm{CO}$; cuando el impulso se intensificó de $56 \mathrm{kPa}$ a 68 $\mathrm{kPa}$, la eficiencia del MCI se redujo y las emisiones aumentaron [34].

En Costa Rica, de forma complementaria al estudio sobre filtros de óxido de calcio y alambrina para incrementar la calidad del biogás de excretas de animal, se evaluó el comportamiento de ungrupo electrógeno basado en MEP, con capacidad de $40 \mathrm{~kW}$ y una conexión de 120/240 V, $60 \mathrm{~Hz}$, trifásico, delta en serie de 4 hilos, operando continuamente con biogás totalmente seco. Esta planta suple durante las 4 horas diarias de mayor demanda la electricidad de la Finca Pecuaria Integrada de EARTH, compuesta por la porqueriza y la lechería, que consumen actualmente $10 \mathrm{~kW} / \mathrm{h}$. Se conectan a la planta eléctrica en las horas pico de demanda: la bomba de vacío del equipo de ordeño, el tanque de enfriamiento de la leche, la bomba de agua, el pulsador de la cerca eléctrica, la picadora de forraje, un trapiche, un refrigerador, una computadora, un ventilador y 10 bombillas. La energía eléctrica adicional generada se puede introducir y utilizar en la red 
eléctrica de la EARTH y mediante transferencia automática, se puede decidir si se utiliza la electricidad del generador o la proveniente del Instituto Costarricense de Electricidad (ICE) [37]. El motor de la planta eléctrica,diseñado para gas natural, fue calibrado para operar con biogás de mínimo de $55 \%$ de $\mathrm{CH}_{4}$ y máximo de 25 ppm de sulfuro de hidrógeno $\left(\mathrm{H}_{2} \mathrm{~S}\right)$, el biogás se suministró a presión entre 7 y $21 \mathrm{kPa}$ con un rango de composición de metano variando entre 61 y $97 \%$, de forma que la proporción promedio de metano en el biogás fue de 79,3\% y su poder calorífico se estimó de $7372 \mathrm{kcal} / \mathrm{m}^{3}$.

Para un factor de utilización de planta del 35\%, el consumo promedio de biogás fue de $16 \mathrm{~m}^{3} / \mathrm{h}$ con una electricidad promedio consumida de 19 $\mathrm{kW} / \mathrm{h}$, dando una eficiencia de generación del $7 \%$ $\mathrm{y}$ una relación de $2 \mathrm{~m}^{3}$ de biogás por cada $\mathrm{kW} / \mathrm{h}$ generado [35], [36].

En Colombia, para MCI de baja potencia operando con biogás, se estudió el desempeño de un MEP de 4 tiempos con relación de compresión de 7,5:1 bajo diferentes composiciones de $\mathrm{CH}_{4} \mathrm{y} \mathrm{CO}_{2}$. Se evaluó la potencia al freno, la eficiencia y las emisiones variando el avance de encendido, donde se observó que al disminuir el porcentaje de $\mathrm{CH} 4$ en el biogás se presenta una reducción del rango de velocidad de rotación del motor y se requiere de un aumento del ángulo de avance de encendido, que a su vez varía en función de la velocidad de rotación. También se apreció una reducción drástica de la potencia máxima, del torque máximo y de la eficiencia respecto al uso de gasolina fósil, para parámetros de operación óptimos del motor a las diferentes velocidades de rotación.

Adicionalmente dicho estudio recomienda que al utilizar un gas pobre de forma permanente en motores de características similares debe aumentarse la relación de compresión, que permitiría obtener una mayor potencia y así un mejor desempeño con gas, pero con la desventaja de no poder em- plear nuevamente gasolina, ya que la mayor relación de compresión implica el uso de un combustible de mayor octanaje para evitar la detonación en el motor. Propone finalmente el estudio del MEP operando con diferentes mezclas de biogás mediante la modificación de parámetros de ajuste del motor para así mejorar la curva de rendimiento [38].

Los MEP diseñados para gasolina y que operan con biogases, tienden a ser menos eficientes al aumentarse el porcentaje de dióxido de carbono en el biogás, ya que el $\mathrm{CO}_{2}$ admitido en el combustible no es reactivo, ocupa espacio que impide el suministro de más combustible reactivo y adicionalmente actúa como moderador de la combustión lo que disminuye el poder calorífico del combustible.Para estos motores los conductos de admisión fueron dimensionados para el funcionamiento con gasolina y el exceso de aire en operación estable se acerca al 1,1, lo que ocasiona que la mezcla de biogás-aire alcance una energía total durante la combustión menor que en la operación con gasolina. Con la disminución de la energía total alcanzada en la mezcla después de la combustión, la potencia producida disminuye en la misma proporción. Un biogás de $60 \% \mathrm{CH}_{4}$ cuyo poder calorífico es de $25 \mathrm{MJ} / \mathrm{m}^{3}$ se considera un gas pobre de media capacidad y causa una reducción de potencia alrededor del 20\% (metano purificado o gas natural 10\%, GLP 5\%).

La potencia y la eficiencia volumétrica del MCI para un mismo régimen dependen en gran medida de la composición del biogás, siendo mayor en operación con biogases de alta composición de metano que con composiciones débiles de metano. Por ejemplo, la potencia producida y la eficiencia volumétrica obtenida en un MEP, son mayores operando con biogás de $70 \%$ de $\mathrm{CH}_{4}$ que operando con biogás de $50 \%$ de $\mathrm{CH}_{4}$, puesto que el primero tiene un mayor poder calorífico y menor cantidad de moderador de la combustión $\left(\mathrm{CO}_{2}\right)$. 
La reducción de potencia en los MEP (afectación principal) debe preverse desde el mismo momento en que se seleccione el motor apropiado a una clase de potencia, para una aplicación dada y con una demanda de potencia especificada.

En los MEP el control de la potencia y velocidad se realiza por variación del suministro de mezcla aire/combustible, mediante el accionamiento de la válvula de mariposa situada entre el dispositivo mezclador y la entrada del motor. El cierre de la válvula de mariposa produce una caída de presión (efecto de estrangulamiento) en el flujo de mezcla, por lo cual el llenado del cilindro se da a baja presión lo que ocasiona una menor cantidad de mezcla aire/combustible con respecto a una masa y energía de referencia. Como resultado la potencia producida, la presión media efectiva y la eficiencia presentan una disminución en operación controlada (carga parcial) donde la disminución de la eficiencia se observa por el aumento del consumo específico de combustible.

Para compensar los efectos antes mencionados, el motor debería mantenerse operando a velocidad media pero con mariposa abierta, lo que requiere de una combinación apropiada a los requerimientos de velocidad y potencia del equipo movido por el motor [28].

\section{EFECTO DE LA RELACIÓN DE COMPRESIÓN SOBRE EL RENDIMIENTO Y LAS EMISIONES DE LOS MOTORES DE COMBUSTIÓN INTERNA (MCI)}

India ha realizado investigaciones sobre la combustión del biogás en cuanto a sus aplicaciones y sus efectos sobre el medio ambiente. Se estudió la influencia de la relación de compresión sobre el rendimiento y la combustión del biogás como combustible para un MEP. El motor se operó a 1500 rpm con una apertura del acelerador entre $25 \%$ y $100 \%$. Las pruebas cubrieron una amplia gama de aire-combustible, aplicando una serie de relaciones de compresión. Además, se realizó una sincronización de la chispa con la cual se estableció un MBT (mejor par de trabajo), lo cual incide notablemente en la eficiencia y desempeño mecánico del motor. La investigación describió el rendimiento, la cantidad de emisiones y el curso de la combustión al variar la relación de compresión, lo que permitió hacer comparaciones de eficiencia. Se encontró a partir de los resultados que cuanto mayor sea la relación de compresión, mayor será el rendimiento térmico. Cuando esta se encuentra por encima de un valor crítico de 13:1, la potencia de freno y la eficiencia térmica se incrementan levemente; se realizó la medición de emisiones de $\mathrm{NOx}, \mathrm{HC}$, observándose un aumento. El valor de la potencia y eficiencia térmica alcanzó sus valores más altos con relaciones de compresión de 13:01 a 15:01 y una relación aire-combustible para el biogás entre 1,08 y 0,95 . Bajo estas condiciones, las emisiones de $\mathrm{HC}$ y $\mathrm{CO}$ fueron bajas, sin embargo, los valores de NOx fueron altos. La sincronización de la chispa MBT se retrasa con el aumento de la relación de compresión, ya que la mezcla tiende a hacerse pobre. Se pudo apreciar que el pico de presión es mayor con relaciones altas siendo notorio un aumento en el nivel de liberación de calor [39].

Se puede concluir que el aumento de la relación de compresión es fundamental a la hora de mejorar el rendimiento y reducir las emisiones del biogás, ya que existe una mejora apreciable en la eficiencia térmica y en la potencia de freno. Se obtuvieron mejoras de hasta el $10 \%$ en la potencia del motor y un aumento del rendimiento del $23 \%$ al $26,8 \%$ para efectos de esta prueba, esto también afecta las condiciones de comportamiento, ya que se observó una reducción en el retardo de la ignición [17]. 


\section{INFLUENCIA DE LA CONCENTRACIÓN DE METANO EN EL BIOGÁS}

El Dr. Porpatham del Instituto de Tecnología de Madras ha realizado varias investigaciones respecto al comportamiento y caracterización de MCI que trabajan con biogás. Uno de sus estudios trata sobre la reducción en la concentración de $\mathrm{CO}_{2}$, las emisiones de combustión y avance de encendido analizando su influencia en el rendimiento. La investigación se realizó sobre un MEP, el cual se sometió a un depurador de agua de cal que se utiliza para reducir el dióxido de carbono en rangos de 41,30 y $20 \%$ para el biogás. Las pruebas se realizaron a una velocidad constante de $1500 \mathrm{rpm}$ y relación de compresión de 13:1. La reducción del nivel de $\mathrm{CO}_{2}$ produjo una significativa mejora en el rendimiento y la reducción de las emisiones de $\mathrm{HC}$, en particular con mezclas pobres. Las tasas de liberación de calor mostraron una mejor combustión, lo que manifestó un aumento en la eficiencia térmica. Se evidenció una reducción en el nivel $\mathrm{CO}_{2}$ del $10 \%$ que parecía ser suficiente para reducir los niveles de $\mathrm{HC}$ y los niveles de NO, pero su disminución resultóno ser considerable [40]. La eliminación de $\mathrm{CO}_{2}$ muestra una composición del biogás con alta concentración de metano y oxígeno, por lo tanto conduce a la rápida combustión y también a mayores niveles de potencia. En China se hizo una investigación en la cual se realizaron pruebas para estudiar la relación de compresión variable de un MEP Ricardo E6 monocilíndrico, formado a partir de diferentes mezclas de gas natural y $\mathrm{CO}_{2}$. La fracciónde $\mathrm{CO}_{2}$ en el biogás simulado fue cambiando de $0 \%$ a $40 \%$ en volumen, para cubrir el intervalo típicamente encontrado en las fuentes de biogás proveniente de biodigestores comunes. Las pruebas cubrieron varios rangos de composición y mezclas ricas en combustible a 4 regímenes de velocidad. Los resultados medidos se dan para potencia, temperatura de escape, eficiencia térmica y fracciones molares de monóxido de carbono de las emisiones reguladas $\mathrm{CO}$, NOx y emisiones de $\mathrm{HC}$ en los gases de escape. Los resultados experimentales indicaron que la presencia de $\mathrm{CO}_{2}$ puede reducir las emisiones de NOx, pero con depresiones más bajas del cilindro, la potencia del motor y la eficiencia térmica se reducen y el nivel de $\mathrm{HC}$ sin quemar se incrementa. Los datos medidos, sin embargo, sugieren que es posible aumentar significativamente la relación de compresión como un medio eficaz para la mejora en el rendimiento del MCI cuando el $\mathrm{CO}_{2}$ está presente. Una notable observación es que si bien suele aumentar las emisiones de NOx y HC, el primero se ve compensado por el $\mathrm{CO}_{2}$ contenido en el biogás.

Como conclusiones relevantes se observa que el $\mathrm{CO}_{2}$ presente en el biogás influye en el funcionamiento del motor, en especial en cuanto a la reducción de las emisiones de NOx y en el aumento en la relación de compresión. También se reduce la presión del cilindro, la potencia y rendimiento térmico son reducidos en un $3 \%$ cuando la fracción de $\mathrm{CO}_{2}$ aumenta a $40 \%$ y las emisiones de $\mathrm{HC}$ totales se incrementaron también con la presencia de $\mathrm{CO}_{2}$ en el combustible [41].

\section{CONCLUSIONES}

El uso de biogases en MCI representa una ventaja significativa en el control del cambio climático por la mitigación de gases de efecto invernadero que afectan el medio ambiente; sin embargo, se hace necesario en términos sociales, ambientales y económicos lograr un desempeño óptimo de los MEP y MEC alimentados con biogás, de modo que se llegue a una caracterización de los parámetros de ajuste que permitan su operación en un entorno controlado [42], que minimice los costos de operación y brinde un impacto positivo en el rendimiento y emisiones finales.

Las investigaciones están dirigidas a realizar modificaciones en el sistema de mezcla aire combustible, rangos de composición de biogás en cuanto 
a cantidad de $\mathrm{CH}_{4}$ y $\mathrm{CO}_{2}$, la presión de suministro de combustible, la relación aire combustible, la adición de porcentajes controlados de $\mathrm{H}_{2}$ inferiores al $15 \%$, así como el poder controlar variables de prueba en cuanto a porcentaje de carga, velocidad de rotación y par efectivo del motor [43].
Esta tendencia mundial hacia la caracterización de parámetros de comportamiento ha permitido avanzar en el conocimiento de los MCI operando con biogás tanto puro como mezclado y en el mejoramiento de su eficiencia de operación [44].

\section{REFERENCIAS}

[1] Organization of the United Nations, Status of Ratifications. Last modified on: New Zealand 14 January 2009.

[2] Kyoto Protocol, Cambio Climático: Situación Actual y Perspectivas, WWF/adena. Gran Vía de San Francisco, 8-D. 28005 Madrid. España, Naciones Unidas 2008.

[3] FAO. Manual de Biogás. Remoción de Barreras para la Electrificación Rural con Energías Renovables, Proyecto CHI/00/ G32, Chile,2011.

[4] R. Souza,"Performance of a motogenerator adapted for biogas",Cienc. agrotec.,Lavras, vol. 34, no. 1, pp. 190-195, jan./fev,Brasil, 2010.

[5] Kyoto Protocol, Status of Ratificación, Organización de las Naciones Unidas. Convención Marco de las Naciones Unidas sobre el Cambio Climático, 14 de enero de 2009.

[6] A. Fankhauser and A. Moser, Estudio sobre la adecuación del biogás como combustible para los tractores agrícolas. Tanikon, Switzerland: FAT Publications, no. $18,1983$.

[7] Buttner, S. Unidad de tractor en biogás - Conversión y primeras experiencias operacionales,no.6, KTBL, Darmstadt. FRG, (German) 1982.
[8] Londrina-PR, Embrater. Encontro de Biogas Automotivo pare Empresa Rural, Brazilia, Brazil, Coletanea dos trabalhosa presentados, 1984.

[9] L. Sasse Ludwig, GATE/. Biogas Plantofs Deutsches Zentrum fur Entwicklungstechnologien. Deutsche Gesellschaft fur Technische Zusammenarbeit (GTZ), 1988.

[10] Borda/GTZ. Biogas Handbuch, In Eggelingetál., Bremen, FRG. (German), 1979.

[11] Oekotop/GATE, Guía práctica para las plantas de biogás en la producción animal, In U. Werner et ál. Eschborn, FRG, Viewg (German), 1986.

[12] L. Sasse et ál, GTZ. Production and Utilization of Biogas in Rural Areas of Industrialized and Developing. Countries,Hohlfield, Eschborn, FRG, 1986.

[13] M. Mwandosya, and S. Lwakabamba. Development in Eastern and Southern Africa, Vol.I, II, Arusha, Tanzania, 1983.

[14] M.K. Kulkarni. Dual Fuel Biogas Engines. Commonwealth Regional (Asia/Pacific) Rural Technology Programme, Bombay, India, 1980.

[15] Cao Zexi, Application of Biogas on Farm Internal Combustion Engine, Provincial 
Agricultural Machinery Research Institute of Sichuan, China, 1982.

[16] S. Khuntia, A. Parida, and N.Vibhuti, Downsizing Technology for Rural Development, Indian Institute of Metals, Bhubaneswar Chapter, Regional Research Laboratory (Bhubaneswar, India) 2005.

[17] R. Omid. Literature review and road map for using biogas in internal combustion engines, University of Stavanger, Norway, May 2011.

[18] J.A. Viquez, Generación eléctrica con biogás. Una alternativa de ahorro eléctrico para granjas lecheras y porcinas. AGROMEC. VIOGAZ S. A. Especialistas en Tecnología de Biogás, 2010.

[19] Anaerobic, Degestion Advise AnaerobicDigestion technology. [Online]. Available: http://www.anaerobic-digestion-advise. com

[20] J.M.Desantes, Experiments on the influence of intake conditions on local instantaneous heat flux in reciprocating internal combustion engines, Valencia: Universidad Politécnica de Valencia, 2010.

[21] J. Retamal y J. Marchant, Cogeneradores para Aplicaciones Agroindustriales y Agropecuarias, Biotamb S.A. Santiago de Chile.

[22] J. Canavate Ortiz, Diesel Engine Modification to operate on Biogas, Transactions of the ASAE, 1981.

[23] K. Mitzlaff and M.H. Mkumbwa, Use of Biogas as an Alternative Fuel in Stationary Diesel Engines, Tanzania: University of Dar-es-Salaam, 1985.
[24] K. Nagar, Handbook on biogasand it $\square$ fs Applications, New Delhi: National Institute of Industrial Research, 2007.

[25] Técnicas Energéticas, Aplicaciones del Biogás. Buenos Aires: Universidad de Buenos Aires, Facultad de Ingeniería. [enlínea]. Disponible: www.materias.fi.uba.ar.

[26] A.Hartmann, Anaerobic Digestion Technology. Course on Energy in Agriculture: General Aspects and Minihydrooh, Bologna, 1989.

[27] M. Kaltschmitt, and H.Hartmann, Energieaus Biomasse. Grundlagen, Techniken und Verfahren, Springer-Verlag. Berlin.

[28] S. Mihic, Biogas fuel for internal combustion engines, Serbia y Montenegro: University of Novi Sad, Faculty of engineering, 2004.

[29] I.D. Bedoya, Efecto del grado de carga y la cantidad del combustible piloto en el comportamiento mecánico ambiental de un motor dual diesel-biogás para generación de electricidad. Medellín: Grupo de Ciencia y Tecnología del Gas y Uso Racional de la Energía, Universidad de Antioquia, 2007.

[30] I.D. Bedoya,"Efecto del grado de carga y la cantidad de combustible teórico", Rev. Fac. Ing. Univ. Antioquia, no. 42, pp. 7993, Diciembre 2007.

[31] Grundfos, Sistemas de dosificación de Gas, GS 141-145 Instrucciones de Manejo, Pfinztal (Sollingen), 2004.

[32] C.D.Rakopoulos, Second-law analyses applied to internal combustion engines operation, National Technical University of Athens, Greece, October 2005. 


\section{revisión}

[33] J, Caton, The thermodynamic characteristics of high efficiency, internal-combustion engines. Texas A\&M University, Dallas, 2012.

[34] S.Siripornakarachai, and T.Sucharitakul, Modification and tuning of Diesel bus engine for biogas electricity production, Thailand: Department of Mechanical Engineering, Faculty of Engineering, Chiang Mai University, 2007.

[35] R. Quesada y N. Salas, Generación eléctrica a partir de biogás. Las Mercedes de Guacimo, Limón. Costa Rica: Universidad EARTH, 2007.

[36] C, Hernández,Utilización de Biogás para uso en Motores de Combustión Interna, Serie de documentos técnicos,Las Mercedes de Guacimo, Limón. Costa Rica: Universidad EARTH, 2004.

[37] O. Nacke, and M.Helm, Biogas von A bis Z. Borsig Energy Gmb H, Oberhausen, Serbia, Novi Sad, 2001.

[38] J. Mantilla, Evaluación experimental de un motor encendido por chispa que utiliza biogás como combustible. Bogotá: Universidad Nacional de Colombia, Redalyc, 2008.

[39] E.Porpatham, Effect of compression ratio on the performance and combustion of a biogas fuelled spark ignition engine, India: Science Direct, Indian Institute of Technology Madras, 2011.

[40] E.Porpatham, Investigation on the effect of concentration of methane in biogas when used as a fuel for a spark ignition engine, India: Science Direct, Indian Institute of Technology Madras, 2007.

[41] J. Chulyoung, Generating efficiency and emissions of a spark-ignition gas engine generator fuelled with biogashydrogen, Blends School of Mechanical Engineering, Yonsei University, 134 Sinchon-dong, Seodaemun-gu, Seoul 120-749, Republic of Korea.

[42] K. Cupial, and G.Katolik, An anomaly of combustion process in the biogas internal combustion engine with automatic control of discharge energy, Czestochowa University of Technology Institute of Internal Combustion Engines and Control Engineering, 2001.

[43] F.E.Sierra, Tecnologías para el Aprovechamiento de los Biocombustibles, Bogotá: Universidad Nacional de Colombia, 2008.

[44] H. Jingdang, Assessment of simulated biogas as a fuel for the spark ignition, Departament of Mechanical and Electrical Engineering, Fujian Agricultural University 1998. 